\title{
Topics on the Degenerate Elliptic Differential Operators Far from Self Adjoint Ones
}

\author{
Ali Sameripour* and Ahmad Rezaei \\ Department of Mathematics, Lorestan University, Khorramabad, Iran; asameripour@yahoo.com, \\ Rezaee70@yahoo.com
}

\begin{abstract}
Background: Elliptic differential operators and asymptotic distribution of eigenvaluesof them are discussed in many works. Methods: In this paper we get some new results about an important differential operator on a Hilbert space. Also asymptotic distribution of eigenvalues of this kind of differential operators are proved with new methods that estimation of the resolvent of the operators is used in this paper. Finding: We get some new theorems about the differential operator A.We consider a bounded domain $\Omega$ with smooth boundary in $\mathrm{R}^{\wedge} \mathrm{n}$ define a norm and find the asymptotic distribution of eigenvalues of the operator $(A u)(x)=-\sum_{i, j=1}^{n}\left(p^{2 a}(x) a_{i j}(x) q(x) u_{x_{i}}^{\prime}(x)\right)_{x_{j}}^{\prime}$ in the space $H_{l}=L^{2}(\Omega)^{l}$. Improvement: We improve the method of proving this kind of theorems.
\end{abstract}

Keywords: Asymptotic Distribution, Eigenvalues, Non-SelfAdjoint Elliptic Differential Operator, Resolvent

\section{Introduction}

Let $\Omega$ be a bounded domain with smooth boundary in, $\mathrm{R}^{\mathrm{n}}$ i.e, $\partial \Omega \in C^{\infty}$. We introduced the space $H_{l}=W_{2}^{2}, \alpha(\Omega) \times \ldots \times W_{2}^{2}, \alpha(\Omega)(\ell-$ times $)$ as the space of vector functions $u(x)=\left(u_{1}(x), \ldots, u_{\ell}(x)\right)$ defined on $\Omega$ with finite norm ${ }^{1-5}$ :

$\left|u, W_{2}^{2}, \alpha(\Omega)\right|=\left(\sum_{i=1}^{n} \int \rho^{2 a}(x)\left|u_{x_{i}}^{\prime}(x)\right|_{C^{\prime}}^{2} d x+\int_{\Omega}|u(x)|_{C^{\prime}}^{2}\right)^{1 / 2}$

By $\mathrm{H}_{\ell}$ we denote the closure of $C_{O}^{\infty}(\Omega)^{\ell} i n \mathrm{H}_{\ell}$, for $\ell=1$ we set $\mathrm{H}=\mathrm{H}_{1}$ and $\mathrm{H}=\mathrm{H}_{1}$. In this article, we investigate the asymptotic formula for distribution of the eigenvalues (e.v.) of a non- self adjoint elliptic differential operator A defined by:

$(A u)(x)=-\sum_{i, j=1}^{n}\left(\rho^{2 a}(x) a_{i j}(x) q(x) u_{x_{i}}(x)\right)_{x_{j}}$

in space $\mathrm{H}_{\ell}=L_{2}(\Omega)^{\ell}$ here,

$\rho(x)=\operatorname{dist}\{x, \partial \Omega\}, \alpha \in[1,0), a_{i j}(x)=\overline{a_{i j}(x)}(i, j=1,2, \ldots, n)$,

$a_{i j}(x) \in C^{2} \overline{(\Omega)}(i, j=1,2, \ldots, n), q(x) \in C^{2}\left(\bar{\Omega}, E n d C^{\ell}\right)$,

Further more assume that for, $\forall_{x} \in \bar{\Omega}$, the matrix function $\mathrm{Q}(\mathrm{x})$ has simple eigenvalues $\mu_{1}(x), \ldots, \mu_{\ell}$ arranged in the complex plane in the following way: $\arg \mu_{j}(x)=0(1,2, \ldots, v), \mu_{j}(x) \notin \bar{\phi}(j=v+1, \ldots, \ell)$,

Where $\phi=\{z \in C:|\arg z|<\varphi\}, \varphi \in(0, \pi)$. We also assume that the matrix function $\mathrm{a}_{\mathrm{ij}}(\mathrm{x})$ satisfies the uniformly elliptic condition: i.e., there exist $\mathrm{M}>0$ such that for every $\mathrm{S}=\left(s_{1}, \ldots, S_{n}\right) \in C^{n}, x \in \Omega$ we have:

$|s|^{2} \leq M \Sigma_{i, j=1}^{n} a i j(x) s_{j} \overline{s_{j}}$.

Now for a closed extension of the operator A we need to extend its domain to the:

$$
\begin{aligned}
& D(A)=\left\{u \in H_{\ell} \cap W_{2}^{2}, \operatorname{loc}(\Omega)^{\ell}: \sum_{i, j=1}^{n}\left(\rho^{2 a} a_{i j} u_{x i}\right)_{x i} \in \mathrm{H}_{\ell}\right\}, \\
& \text { Where } W_{2}^{2}, \operatorname{loc}(\Omega)=\left\{u: \sum_{i=0}^{n} \int_{j}\left|u^{(i)}(x)\right|^{2} d x<\infty \text { ojisopensetin } \Omega\right\}
\end{aligned}
$$

Here and in the sequel the value of the function $z \in(-\pi, \pi]$ and $\|T\|$ denotes the norm of the bounded operator $T: \mathrm{H}_{\ell} \rightarrow \mathrm{H}_{\ell}$.

\section{Resolvent Estimate}

Theorem 2.1. Let $S \subset \phi \backslash R_{+}$be some closed sector with vertex at 0 and as in Section 1, we recall that for, $\forall_{x} \in \bar{\Omega}$, the matrix function $\mathrm{q}(\mathrm{x})$ has simple eigenvalues $\mu_{1}(x), \ldots, \mu_{\ell}(x)$ which are different from zero $\arg \mu_{\mathrm{j}}(\mathrm{x})=0$ for $\mathrm{j}=1,2, \ldots, \mathrm{v}$ and 
$\mu_{1} \notin \bar{S}$ forj $=v+1, \ldots, \ell$ Where $\phi\{z \in C:|\arg z|<\varphi\}, \varphi \in(0, \pi)$

(i.e., the eigenvalues $\mu_{1}(x), \ldots, \mu_{v}(x)$ lie on the positive real line insides the angle $\Phi$, and the rest of the eigenvalues $\mu_{v+1}(x), \ldots, \mu_{\ell}(x)$ lie outside of the angle $\Phi$ in view of $S \subset \phi \backslash R_{+}$implies that all the eigenvalues $\mu_{1}(x), \ldots, \mu_{\ell}(x)$ lie on the complex plane and outside of the closed sector )$^{6-8}$. Then the operator has a discrete spectrum and for sufficiently large in modules $\lambda € S$ the inverse operator(A$\lambda \mathrm{I})^{-1}$ exists and is continuous and the following estimate is valid:

$$
\left\|(A-\lambda I)^{-1}\right\| \leq M_{S}|\lambda|^{-1}\left(\lambda \in S,|\lambda| \geq C_{S}\right)
$$

where $\mathrm{M}_{\mathrm{s}}, \mathrm{C}_{\mathrm{s}} \mathrm{CS}$, are sufficiently large numbers. The symbol \|.\| stands for the norm of a bounded operator in $\mathrm{HorH}_{\ell}$.

Proof of Spectral Theorem 2.1. Base on the assumptions, the eigenvalues of the matrix function have $\mathrm{q}(\mathrm{x})$ the following conditions $\mathrm{s}^{9-11}$ :

$\mu_{k}(x) \in C^{2}(\bar{\Omega}), k=1, \ldots, \ell$ such that $\mu_{1}(x), \ldots, \mu_{\ell}(x)$ lie on the positive

real line insides the angle $\Phi$ and the rest of the eigenvalues $\mu_{\mathrm{v}+1}(\mathrm{x}), \ldots, \mu_{\ell}(\mathrm{x})$ lie outside of the angle $\Phi$, then in view of the conditions on the eigenvalues, we defined the operators $\mathrm{P}_{\mathrm{k}}\left(\mathrm{k}=1,2, \ldots\right.$, on $\mathrm{H}=\mathrm{H}^{\prime}$ by

$$
\begin{aligned}
& \left(p_{k y}\right)(x)=-\sum_{i, j=1}^{n}\left(\rho^{2 a}(x) a_{i j}(x) \mu_{k} y_{x i}(x)\right)_{x_{j}} \\
& D\left(P_{k}\right)=\left\{y \in \mathrm{H} \cap W_{2}^{2}, \operatorname{loc}(\Omega): \sum_{i, j=1}^{n}\left(\rho^{2 a} a_{i j} \mu_{k} y_{x_{i}}\right)_{x_{j}} \in \mathrm{H}\right\},
\end{aligned}
$$

Since the eigenvalues $\mu_{v+1}(x), \ldots, \mu_{\ell}(x)$ lie outside of the angle $\Phi$ therefore for $k \in\{v+1, \ldots, \ell\}$, we assume also that there exist distinct numbers: $\psi_{v+1}, \ldots, \psi_{\ell} \in(\varphi, \pi) \cup$ $[-\pi,-\varphi)$, such that. $\arg \mu_{j}(x) \equiv \psi_{j}(j=v+1, \ldots, \ell)$. such that the eigenvalues $\mu_{j}(\mathrm{x})$ lie on the fix rays in the complex plane which the angle between $(\bar{\Omega})$ each two consequent ray are equals to $\frac{\pi}{16}$.

Thus, by this assumption, we construct the nonegative functions, $\varphi_{k_{1}}(x), \ldots, \varphi_{m}(x) \in C^{\infty}$ with the following properties:

$$
\sum_{i=1}^{m} \varphi_{k_{i}}^{2}(x) \equiv 1(x \in \overline{(\Omega)}),\left|\arg \left\{\mu_{k}\left(x_{1}\right) \mu_{k}^{-1}\left(x_{2}\right)\right\}\right|<\frac{\pi}{16}\left(\forall x_{1}, x_{2} \in \sup p \varphi k_{j}\right),
$$

for any $\mathrm{j} \in\{1, \ldots, \mathrm{m}\}$. Let us construct the functions $\mu_{\mathrm{kr}}(\mathrm{x}) \in \mathrm{C}^{2}(\bar{\Omega})$ satisfying these conditions:

$\mu_{k_{r}}(x)=\mu_{k}(x),\left(\forall x \in \sup p \varphi_{k_{r}}\right), \mu_{k_{r}}(x) \notin \phi,\left(\forall_{x} \in(\bar{\Omega})\right)$,

Fork $=v+1, \ldots, \ell$ and $r=1, \ldots, m$ we have

$\left|\arg \left\{\mu_{k_{r}}\left(x_{1}\right) \mu_{k_{r}}^{-1}\left(x_{2}\right)\right\}\right|\left(\forall_{x_{1}},_{x_{2}} \in \phi\right)$
And there exist numbers, $\left|Z_{\mathrm{kr}}\right| \in \mathrm{C}$ such that $\left|Z_{k_{r}}\right|=1$ for $\forall x \in \bar{\Omega}, \lambda \in \bar{\Phi}$ for we have

$C^{\prime} \leq \operatorname{Re}\left\{Z_{k_{r}} \mu_{k_{r}}(x)\right\}, C|\lambda| \leq-\operatorname{Re}\left\{Z_{k_{r}} \lambda\right\}, C^{\prime}>0$.

For $\mathrm{k}=\mathrm{v}+1, \ldots, \ell$ by applying the functions $\mu_{k_{r}}(x)$ as the operators $\mathrm{P}_{\mathrm{k}}$ on $\mathrm{H}=\mathrm{H}_{1}$ above, we defined the operators, $\mathrm{P}_{\mathrm{k}} \mathrm{r}$, on $\mathrm{H}=\mathrm{H}_{1}$ by:

$\left(P_{k y}\right)(x)=-\sum_{i, j=1}^{n}\left(\rho^{2 a}(x) a_{i j}(x) \mu_{k} y_{x i}(x)\right)_{x_{i}}$

$\left.D\left(P_{k}\right)=\left\{y \in \mathrm{H} \cap W_{2}^{2} \operatorname{loc}(\Omega): \sum_{i, j=1}^{n} \rho^{2 a} a_{i j} \mu_{k} y_{x_{i}}\right)_{x_{j}} \in \mathrm{H}\right\}$.

Take $\mathrm{I},(y)=\Sigma_{j}^{n} \int_{\Omega} \rho^{2 a}(x)\left|y_{x_{i}}(x)\right|^{2} d x, \quad$ then from uniformly elliptic condition we have for : $c>0$ :

$c \sum_{i=1}^{n}\left|y_{x_{i}}(x)\right|^{2} \leq \sum_{i, j=1}^{n} a_{i j}(x) y_{x_{i}}(x) \overline{y_{x_{j}}(x)}=<\alpha(x) \nabla_{x} y(x)>C^{n},, p^{\alpha} y_{x_{j}}$

Then for $\in \mathrm{D}\left(P_{k_{r}}\right), \mathrm{k}=\mathrm{v}+1, \ldots, l$ according to we have:

$I(y) \leq M_{1} \operatorname{Re}\left\{Z_{k_{r}} \sum_{i, j=1}^{n}\left(\rho^{\alpha} a_{i j} \mu_{k r} y_{x_{j}}, \rho^{\alpha} y_{x_{j}}\right)\right\}=M_{1} \operatorname{Re}\left\{Z_{k_{r}}\left(P_{k_{r}} y, y\right)\right\}$.

here and in sequel, the symbol (,) denotes the inner product in $H$. Now according to (2.2) for $\lambda \in \bar{\Phi}$ we have: $I(y)+|\lambda||y|_{H}^{2} \leq M_{2} \operatorname{Re}\left(\left(Z_{k_{r}} P_{k_{r}}-\lambda Z_{k_{r}} I\right) y, y\right) \leq M_{2}|y| H\left|\left(P_{-}\left(k_{-} r\right)-\lambda I\right) y\right| H$, $I(y) \leq M_{2}|y| H \mid\left(P_{k_{r}} y_{H^{\prime}}\right.$

in put $\mathrm{y}=\left(P_{k_{r}}-\lambda I\right)^{-1} f \in \mathrm{H}$ then we have: $|\lambda|\left(P_{k_{r}}-\lambda I\right)^{-1} f\left|\leq M_{2}\right|\left(P_{k_{r}}-\lambda I\right)\left(P_{k_{r}}-\lambda I\right)^{-1} f_{H} \mid$ therefore

$\left|\left(P_{k_{r}}-\lambda I\right)^{-1} f\right| \leq M|\lambda|^{-1} f_{H}$ hence $\left\|\left(P_{k_{r}}-\lambda I\right)^{-1}\right\| \leq M|\lambda|^{-1}$ $\left\|\left(P_{k_{r}}-\lambda I\right)^{-1}\right\| \leq M(\lambda \in \bar{\Phi}, \lambda \neq 0)$

In (2.3) we put $y=\left(P_{k_{r}}-\lambda I\right)^{-1} f, f \in H$, , then we will have:

$\left.\left|\rho^{\alpha} \frac{\partial}{\partial_{x_{i}}}\left(P_{k_{r}}-\lambda I\right)^{-1} f\right|_{H}^{2} \leq M|f|_{H} \mid P_{k_{r}}-\lambda I\right)\left.^{-1} f\right|_{H} \leq M|\lambda|^{-1}|f|_{H}^{2},(i=1, \ldots, n)$

for proof (2.5) we have:

$\left|\rho^{\alpha} \frac{\partial}{\partial_{x_{i}}}\left(P_{k_{r}}-\lambda I\right)^{-1} f\right|^{2} H=\int_{\Omega} \rho^{2 a}\left|\frac{\partial}{\partial_{x_{i}}}\left(P_{k_{r}}-\lambda I\right)^{-1}\right|^{2} d x$

$\leq \sum_{i=1}^{n} \int_{\Omega} \rho^{2 a}\left|\frac{\partial}{\partial_{x_{i}}}\left(P_{k_{r}}-\lambda I\right)^{-1} f\right|^{2}$

$=I\left(P_{k_{r}}-\lambda I\right)^{-1} f$ 
i.e. in $\mathrm{I}(\mathrm{y})$ above we put $\mathrm{y}=\left(P_{k_{r}}-\lambda I\right)^{-1} f$; so that we will have:

$\left|\rho^{\alpha} \frac{\partial}{\partial_{x_{i}}}\left(P_{k_{r}}-\lambda I\right)^{-1} f\right|_{H}^{2} \leq I\left(\left(P_{k_{r}}-\lambda I\right)^{-1} f\right)$.

Since by we have $\mathrm{I}(\mathrm{y}) \leq \mathrm{M}_{2}|\mathrm{y}|_{\mathrm{H}}\left|P_{k_{n}} \mathrm{y}\right|_{\mathrm{H}}$ and by (2.4) $\left\|P_{k_{r}}\left(P_{k_{r}}-\lambda I\right)^{-1}\right\| \leq$ therefore if in $\mathrm{I}(\mathrm{y}) y=\left(P_{k_{r}}-\lambda I\right)^{-1} f$ we set then we will have:

$\left.\left|\rho^{\alpha} \frac{\partial}{\partial x_{i}}\left(p_{k_{r}}-\lambda I\right)^{-1} f\right|_{H}^{2} \leq\left(\left(p_{k_{r}}-\lambda I\right)^{-1} f\right) \leq M_{2} \mid\left(p_{k_{r}}-\lambda I\right)^{-1} \| p_{k_{r}}-\lambda I\right)^{-1} f \mid$

$\leq M_{2} M\left|\left(p_{k_{r}}-\lambda I\right)^{-1} f\left\|f\left|\leq M_{3}\right|\left(p_{k_{r}}-\lambda I\right)^{-1} f\right\| f\right|$

$\leq M_{3}\left\|\left(p_{k_{r}}-\lambda I\right)^{-1} f\right\||f|_{H}^{2}$

$\left.\begin{aligned} & \text { i.e. } \\ & \text { implies }\end{aligned} \rho^{\alpha} \frac{\partial}{\partial x_{i}}\left(p_{k_{r}}-\lambda I\right)^{-1} f\right|_{H} ^{2} \leq M_{3} M|\lambda|^{-1}|f|_{H}^{2}$. that proof, i.e.

$\left|\rho^{\alpha} \frac{\partial}{\partial x_{i}}\left(p_{k_{r}}-\lambda I\right)^{-1} f\right|_{H} \leq M|\lambda|^{\frac{-1}{2}}|f|_{H}$

Hence $\left\|\rho^{\alpha} \frac{\partial}{\partial x_{i}}\left(p_{k_{r}}-\lambda I\right)^{-1}\right\|_{H} \leq M|\lambda|^{\frac{-1}{2}}$.

From here, according to Hardy's inequality and (2.5) we conclude that

$\left\|\rho^{2 \alpha-1}\left(p_{k_{r}}-\lambda I\right)^{-1}\right\| \leq M_{3} \sum_{i=1}^{n}\left\|\rho^{\alpha} \frac{\partial}{\partial x_{i}}\left(p_{k_{r}}-\lambda I\right)^{-1}\right\| \leq M|\lambda|^{\frac{-1}{2}}$

Now below we introduce in $\mathrm{H}_{\ell}$ the operator:

$G_{k}(\lambda)=\sum_{r=1}^{n} \varphi_{k_{r}}\left(p_{k_{r}}-\lambda I\right)^{-1} \varphi k_{r},(k=v+1, \ldots, \ell)$

Here $\phi_{\mathrm{kr}}$ is the operator of multiplication by function; $\phi_{\mathrm{kr}}(\mathrm{x}) \mathrm{t}$ is easy to verify that:

$\left(P_{k}-\lambda I\right) G_{k}(\lambda)=I+\rho^{2 a-1}(x) \sum_{r=1}^{n} B_{k_{r}}(x)\left(P_{k_{r}}-\lambda I\right)^{-1} \varphi_{k_{r}}$

$+\rho^{2 a \alpha}(x) \sum_{r=1}^{n} \sum_{r=1}^{m} \gamma k_{i r}(x) \frac{\partial}{\partial x_{i}}\left(P_{k_{r}}-\lambda I\right)^{-1} \varphi_{k_{r}}$,

Where $\beta_{k_{i r}}, \gamma k_{i r}(x) \in L_{\infty}(\Omega),(x \in \Omega)$ bounded functions, . $\operatorname{supp} \boldsymbol{\beta} \mathrm{k}_{\mathrm{P}} \cdot \operatorname{supp} \mathbf{k}_{\mathrm{ir}}(\mathbf{x}) \subset \operatorname{supp} \varphi \mathrm{k}_{\mathrm{r}}$

Applying -then we will have:

$\left\|\left(P_{k}-\lambda I\right) G_{k}(\lambda)-I\right\| \leq M|\lambda|^{\frac{-1}{2}}(\lambda \in \overline{\Phi,|\lambda|} \geq 1)$

Thus for $\mathrm{k}=\mathrm{v}+1, \ldots, \ell$ we have this representation:

$\left(P_{k_{r}}-\lambda\right)^{-1}=G_{k}(\lambda)\left(I+F_{k}(\lambda)\right),\left\|F_{k}(\lambda)\right\| \leq M|\lambda|^{\frac{-1}{2}}\left(\lambda \in \bar{\Phi},|\lambda| \geq C_{0}\right)$
Since

$\mu_{k}(x)=\overline{\mu_{k}(x)}>0,(k=1, \ldots, v, x \in \bar{\Omega})$, from $P_{k}=P_{k}^{*} \geq 0,(k=1, \ldots, v)$.

The above equality are well known from estimate of the resolvent of the operators $\mathrm{P}_{\mathrm{K}}$. Therefore in general for $\mathrm{k}=1, \ldots, \ell$ we will have

$\left\|\left(P_{k_{r}}-\lambda\right)^{-1}\right\| \leq M|\lambda|^{\frac{-1}{2}}$.

We now diagonlize the matrix function $\mathrm{q}_{(\mathrm{x})}$ as follows: $q(x)=U(x) \wedge(x) U^{-1}(x)$, Where $U(x), U^{-1}(x) \in C^{2}\left(\bar{\Omega}\right.$, End $\left.C^{\ell}\right)$

and

$\wedge(x)=\operatorname{diag}\left\{\mu_{1}(x), \ldots, U_{\ell}(x)\right\}$,

$\operatorname{Let} \Gamma(\lambda)=U B(\lambda) U^{-1}$ that the operator $\mathrm{B}(\lambda)$ in the direct sum.

$\mathrm{H}_{\ell}=\mathrm{H} \oplus \ldots \oplus \mathrm{H}(\ell-$ times $)$.

has his representation:

$B(\lambda)=\operatorname{diag}\left\{\left(P_{1}-\lambda I\right)^{-1}, \ldots,\left(P_{\ell}-\lambda I\right)^{-1}\right\}$,

Such, that $\lambda \in \bar{\phi} \backslash R_{+},|\lambda| \geq C_{0},(U u)(x)=U(x) u(x),\left(u \in \mathrm{H}_{\ell}\right)$.

It is easy to verify below equality:

$(A-\lambda I) \Gamma(\lambda)=I+\rho^{2 \alpha-1}(x) q_{0}(x) B(\lambda) U^{-10}+\rho^{2 \alpha}(x) \sum_{i=1}^{n} q_{i}(x) \frac{\partial}{\partial x_{i}} B(\lambda) U^{-1}$,

$q_{i}(x) \in C\left(\bar{\Omega}, E_{n d} C^{\ell}\right), \mathrm{i}=0,1, \ldots, \mathrm{n}$, Applying -we have as in

$(A-\lambda I)^{-1}=\Gamma(\lambda)(I+F(\lambda))$

$\|F(\lambda)\| \leq M_{\psi}|\lambda|^{\frac{-1}{2}}\left(\lambda \in \Phi_{\psi},|\lambda| \geq C_{s}\right)$.

Consequently,

$\|\left(A-\lambda I^{-1} \| \leq M_{\psi}|\lambda|^{\frac{-1}{2}}\left(\lambda \in S,|\lambda| \geq C_{S}\right)\right.$.

The proof of Theorem 2.1 is now complete.

\section{Vanishing Limit Arguments}

Theorem 3.1; for sufficiently large in modulus, $\lambda \in \phi_{\Psi}$ where;

$\Phi_{\psi}=\{z \in C: \psi \leq|\arg z| \leq \varphi\}, \psi \in(0, \varphi), \varphi \in(0 . \pi)$

The operator $\mathrm{A}$ has discrete spectrum and the operator A has a finite number of (e.v.) in every set $\phi_{\Psi,} \Psi>$ 
0 of the form, (2.1), therefore we will prove this relation $\lim _{j \rightarrow \infty} \arg \lambda_{j}=0$.

Proof of Theorem 3.1. From the definition of the operator A and from the extension of its domain in the set $^{10,12}$ :

$$
D(A)=\left\{u \in \mathrm{H}_{\ell} \cap W_{2},{ }^{2} \operatorname{loc}(\Omega)^{\ell}: \sum_{i, j=1}^{n} \frac{\partial}{\partial x_{j}}\left(\rho^{2 a} a_{i j} \frac{\partial u}{\partial_{x_{i}}} \in \mathrm{H}_{\ell}\right\}\right.
$$

We know that $D(A) \subset W_{2}^{2}, \alpha(\Omega)^{\ell}$, and so from the compactness of the imbedding of $\left\{\mathrm{H}_{\ell} \cap W_{2}^{2}, \operatorname{loc}(\Omega)^{\ell}\right\} \subset \mathrm{H}_{\ell}$, , and since for $0<\alpha<1$ we know that $\mathrm{A}: \mathrm{D}(\mathrm{A}) \rightarrow \mathrm{H}_{l}$ is compact operator on the Hilbert spaces and since the com- pact operators on the Hilbert spaces have discrete spectrum, therefore the operatorhas discrete spectrum. Next for proof, $\lim _{j \rightarrow \infty} \arg \lambda_{j}=0$, let the sequence of eigenvalues of the operator $\mathrm{A}$ in the angle $\Phi=\{\mathrm{z} \in \mathrm{c}|\arg | \leq \varphi\}, \varphi \in(0, \pi)$ be denoted by $\left\{\lambda_{\mathrm{i}}\right\}$ enumerated in the nondecreasing order of their absolute values and taking into account their multiplicities. Then based on the proof of the theorem 2.1 it conclude that the operator has a finite number of eigenvalues in the set $\Phi_{\psi}, \Psi>0$ let A be a given operator. Denote by $\lambda_{1} \lambda_{2}, \ldots$ the eigenvalues of $\mathrm{A}$ belonging to the following angle;

$\Phi=\{Z \in C:|\arg |<\varphi\}: \varphi \in(0, \pi)$.

And assume that $\lambda_{j} \rightarrow \infty(j \rightarrow \infty)$.

If for every closed sector $S \subset \overline{(\Phi)} \backslash \mathrm{R}_{+}$with origin at zero, there exists a number $C(S)>0$, such that for the inverse $(\mathrm{T}-\lambda \mathrm{I})^{-1}$ exists and is continuous, then the following relation holds; $\lambda_{\mathrm{J}} \rightarrow 0(\mathrm{~J}-\infty)$.

\section{Asymptotic Distribution of Eigenvalues}

Let denotes the number of eigenvalues of the operator located inside the angle $\mathrm{S}$ and does not exceeding the number .i.e., $N(t)=c$ ar $d\left\{j:\left|\lambda_{j}\right| \leq t\right\}, t>0$, then by the assumptions of Section 1, we have the following asymptotic distribution of eigenvalues of the operator in case weak degeneracy.

Theorem 4.1. If $\alpha \in[0,1),<\frac{1}{n}$ then we have $N(t) \sim(2 \pi)^{-n} u_{n} t^{\frac{n}{2}} \sum_{k=1}^{v} \int_{\Omega} \rho^{-n \alpha}(x) \mu_{k}^{\frac{-n}{2}}(x)\left(\operatorname{det}(a(x))^{\frac{-1}{2}} d x\right.$,

Where $\mathrm{v}$ denotes the volume of the unit ball in $R^{n}$ and $\alpha(x)=\left(a_{i j}(x)\right)_{i, j=1}$.

Proof of theorem 4.1.For every natural number S, such that

$S \in N, S>\omega=\max \left\{\frac{n}{2}, \frac{n-1}{2-2 \alpha}\right\}, S \in(\omega, \omega+1]$

We will have:

$$
\left|\operatorname{tr}(A-\lambda I)^{-S}-\operatorname{tr} U B(\lambda) U^{-1}\right| \leq M|\lambda|^{\frac{-1}{2}}|\Gamma(\lambda)|_{S}^{S}
$$

Where the symbols tr, $1,1_{s}$ denote the trace of a trace-class operators and the $\sigma_{\mathrm{s}}$-norm of the operator respectively ${ }^{4}$.

By using the representation of the operator $B(\lambda)$ we obtain:

$\operatorname{tr} U B(\lambda)^{S} U^{-1}=\operatorname{tr} B(\lambda)^{S}=\operatorname{tr} B_{s}(\lambda)$
WhereB $_{s}(\lambda)=\operatorname{diag}\left\{\left(P_{1}-\lambda I\right)^{-s}, \ldots,\left(P_{\ell}-\lambda I\right)^{-s}\right\}$.

If we estimate $|\Gamma(s)|_{s}^{s}$ by using and we obtain this relation:

$\left|\sum_{i+1}^{+\infty}\left(\lambda_{i}-\lambda\right)^{-s}-\sum_{i+1}^{\ell} \sum_{i+1}^{+\infty}\left(\lambda_{i j}-\lambda\right)^{-s}\right| \leq M_{\psi}|\lambda|^{\omega-S-\frac{1}{4}},\left(\lambda \in \phi_{\psi},|\lambda| \geq C_{\psi}\right)$

Where $\lambda_{\mathrm{I}} \lambda_{2}, \ldots, \lambda_{1 j}, \lambda_{2 j}, \ldots$ are denoted respectively the $\left(\mathrm{e}, \mathrm{v}\right.$ ) of the operators $\mathrm{A}$ and $\mathrm{P}_{\mathrm{r}}$. Notice that according to , there are finite number of of the using the counter integral method in the same way as in ${ }^{4,5}$ for $\omega \neq 1,2, \ldots$ we can again obtain like the above relation by counter integral: i.e.

$\sum_{i=1}^{+\infty}\left(\lambda_{i}+\right)^{-s}=\sum_{j=1}^{v} \sum_{i=1}^{+\infty}\left(\lambda_{i j}+\tau\right)^{-s}+0\left(\tau^{\omega-s-\frac{1}{4}}\right), \tau \rightarrow \infty$.

From here and from (2) keeping in mind that $\lambda_{\mathrm{ij}}>0$ (for $i=1,2, \ldots, j=1, \ldots, v$ ). Now it is easy to establish the asymptotical formula:

$\int_{0}^{+\infty} \frac{d N(t)}{(t+\tau)^{s}} \sim \sum_{j=1}^{v} \sum_{i=1}^{v} \int_{0}^{+\infty} \frac{d N_{i}(t)}{(t+\tau)^{s}}, \tau \rightarrow+\infty$

where $N_{i}(t)=c \arg d\left\{j: \lambda_{i j} \leq t\right\}, i=1, \ldots, v$ which are well known asympotical formulas for functions- $\mathrm{N}_{\mathrm{I}}(\mathrm{t}$ ) (see for example ${ }^{6}$, after apply of M. V. Keldish's theorem of Tauberian-type we establish the assertation of Theorem 1,4 in applying the multi dimensional Taubrian Theorems ${ }^{7}$.)

\section{Conclusion}

In this paper we get some new results about an important differential operator on a Hilbert space. Also asymptotic distribution of eigenvalues of this kind of differential operators are proved with new methods that estimation of the resolvent of the operators is used in this paper. 


\section{References}

1. Boimatov KKh, Kostychenko AG. Distribution of eigenvalues of second-order non-selfadjoint differential operators. (Russian) Vest. Moskov Univ. Ser. I Mat. Mekh1990; 3:24-31.

2. Boimatov KKh, Kostychenko AG. The spectral asymptotics of non-selfadjoint elliptic systems of differential operators in bounded domains. MatemSbornik. 1990; 181(12):167893.

3. Boimatov KKh. Asymptotic of the spectrum of non-self-adjoint systems of second-order differential operators. Mat Zametki. 1992 Apr; 51(4):33-7.

4. Boimatov KKh,Seddighi K. On some spectra properties of ordinary differential operators generated by noncoercive forms, Dokl Akad Nauk Rossyi; 1996.

5. Boimatov KKh. Spectral asymptotics of nonselfadjoint degenerate elliptic systems of differential operators Dokl Akad Nauk Rossyi.1993; 330(6):545-53.

6. Kornienko D. On the theory of the spectrum of $2 \times 2$ hyper- bolic systems of the second order. Indian Journal of Science and Technology. 2015 Nov; 8(29):245-53.

7. Kato T. Perturbation theory for linear operators. New York:, Springer; 1966.

8. Naymark MA. Linear differential operators. Moscow: Nauka; 1969.

9. Sameripour A, Seddigh K. Distribution of the eigenvalues non-self-adjoint elliptic systems that degenerated on the boundary of domain. Mat Zametki.1997; 61(3):463-7.

10. Sameripour A, Seddigh K. On the spectral properties of generalized non-selfadjoint elliptic systems of differential operators degenerated on the boundary of domain. Bull Iranian Math Soc. 1998 Jan; 24(1):15-32.

11. Sridhar KP, Muralidharan D. Optimal hamming distance model for crypto cores against side channel threats. Indian Journal of Science and Technology. 2014 Apr; 7(4S): 28-33.

12. Shkalikov AA. Tauberian type theorems on the distribution of zeros of holomorphic functions. Matem Sbornik. 1984; 23(3):337-47.perspectives. 